\title{
Vingt ans de travaux scientifiques sur les réseaux et la mobilité ferroviaires
}

Twenty Years of Research on Railway Networks and Mobility

\section{Etienne Auphan et Gabriel Dupuy}

\section{OpenEdition}

\section{Journals}

Édition électronique

URL : https://journals.openedition.org/rhcf/852

DOI : $10.4000 /$ rhcf.852

\section{Éditeur}

Rails \& histoire

Édition imprimée

Date de publication : 1 juin 2008

Pagination : 95-101

ISSN : 0996-9403

Référence électronique

Etienne Auphan et Gabriel Dupuy, « Vingt ans de travaux scientifiques sur les réseaux et la mobilité ferroviaires », Revue d'histoire des chemins de fer [En ligne], 39 | 2008, mis en ligne le 01 juin 2011, consulté le 22 avril 2022. URL : http://journals.openedition.org/rhcf/852 ; DOI : https://doi.org/ $10.4000 /$ rhcf.852 
Etienne AUPHAN et Gabriel DUPUY

\section{Vingt ans de travaux scientifiques sur les réseaux et la mobilité ferroviaires}

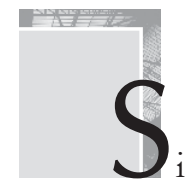

$\mathrm{i}$ on en juge par les articles parus au fil des numéros de la Revue d'histoire des chemins de fer, et par les travaux universitaires soutenus par l'association, la contribution de l'AHICF à la connaissance de l'histoire du chemin de fer à travers les réseaux et la mobilité procède de trois approches principales : la mise en place et la configuration des réseaux, la politique commerciale des compagnies ferroviaires et l'impact du chemin de fer sur la ville et le développement régional.

La mise en place et la configuration des réseaux est abordée quelle que soit leur nature : national principal (par exemple Paris-Rouen-Le Havre) ${ }^{1}$, régional (la Compagnie des Charentes), chemins de fer secondaires départementaux (le Var) ${ }^{2}$, ou urbains (la RATP), et même plus récemment le réseau à grande vitesse. D’une manière assez logique, cette «approche réseau » se veut la base de la recherche en histoire des chemins de fer. Très nombreux sont les travaux qui retracent la mise en place du réseau ferré dans divers territoires : en

1- [58]. Les numéros entre crochets renvoient aux travaux universitaires soutenus par l'AHICF dont la liste est publiée p. 330.

2- [79]. 
Charente, en Haute-Vienne ou au nord-est de Lyon par exemple ${ }^{3}$. Ce thème est précisément celui qui a fait l'objet de la première journée scientifique de l'AHICF en 1989. Il s'agissait d'un premier cadrage du concept de réseau, depuis la conception (références à la théorie de Léon Lalanne; conception du réseau à grande vitesse national et international) jusqu'à son utilisation au regard de l'accessibilité des villes ou des lieux urbains à Paris ou à Lyon en passant par leur configuration analysée au travers de la théorie des graphes. Le réseau à grande vitesse lui-même n'a pas été en reste puisque sa genèse a fait l'objet d'un colloque spécifique en 1994. Si on en laisse de côté l'approche innovation, très développée et retenue par ailleurs dans un autre domaine de ce colloque, l'approche réseau n'en est pas moins très présente ${ }^{5}$.

À l'opposé, les réseaux secondaires ont fait l'objet de nombreux travaux, à commencer par les nombreuses et classiques monographies qui ont porté, entre autres, sur les chemins de fer départementaux du Calvados, de la Drôme, de la Dordogne, du Pas-de-Calais ou de la Gironde ${ }^{6}$. D'autres ont concerné le Réseau Breton, les Landes de Gascogne, l'Est de Lyon ou NiceDigne et même le réseau de tramways du Nord ${ }^{7}$. Plus globalement, les chemins de fer secondaires ont été le thème du neuvième colloque de l'AHICF en $2001^{8}$. Outre un rappel du cadre général dans lequel ils se sont développés, plusieurs monographies y ont été présentées, notamment sur l'Indre, l'Hérault, la Haute-Saône, le Lot-et-Garonne, le Tarn, le Haut-Beaujolais et l'Alsace. Mais c'est surtout l'immense travail de Maurice Wolkowitsch paru en 2004 et intitulé Le Siècle des chemins de fer secondaires en France 1865-1963 qui fera la synthèse magistrale tant attendue sur ce sujet même si ce dernier a fait l'objet de nombreuses monographies plus ou moins nourries antérieurement ${ }^{9}$. Enfin, il ne faut pas oublier les réseaux ferrés urbains comme ceux de Paris et de Lyon $(\mathrm{OTL})^{10}$.

Les chemins de fer d'outre-mer, particulièrement en Afrique, mais aussi dans les îles de l'océan Indien et en Asie, n'ont pas été laissés de côté puisqu'ils ont fait l'objet du thème de la quatrième journée scientifique de l'AHICF en

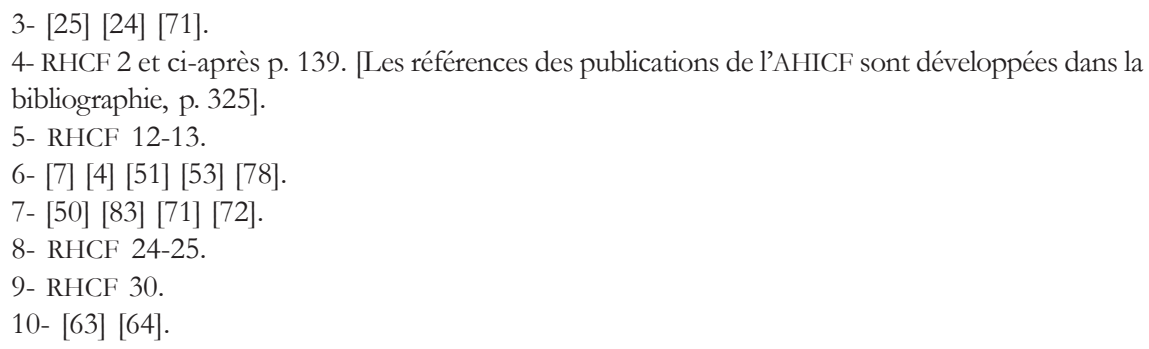


$1991^{11}$. Au regard des réseaux perçus à travers les acteurs économiques et les promoteurs, on peut signaler les études sur des chemins de fer tunisiens, ceux de la Réunion, d'Indochine, de Madagascar, et même sur le Jaffa-Jérusalem. En dehors de ce colloque, d'autres travaux ont porté sur le Transsaharien, l'Abidjan-Ouagadougou et plus généralement sur les chemins de fer de l'Afrique intertropicale ${ }^{12}$. D'une manière moins classique, on s'est interrogé sur le rôle de l'un des grands acteurs industriels dans la mise en valeur de l'Empire à travers la Compagnie des Batignolles pour le Congo-Océan.

Mais un réseau de transport n'offre pas d'intérêt s'il n'est pas accessible aux voyageurs ou aux marchandises. D'où la troisième journée scientifique de l'AHICF : «Les chemins de fer dans la ville » qui a porté sur les points d'accès au réseau que sont les gares urbaines ${ }^{13}$. Ce point est abordé en premier lieu sous l'angle classique de l'histoire de l'art et de l'architecture (par exemple pour la gare d'Orsay à Paris) et de l'urbanisme (Paris, Nancy, Lyon, Lille ou Marseille). Plus originale est l'approche par laquelle est analysée l'influence des chemins de fer sur les architectes au XIX ${ }^{e}$ siècle, ou encore par laquelle sont recherchés les principes de conception des gares. Mais la gare urbaine est soumise au poids des ans et sa fonction dans la cité évolue avec le temps. C'est tout le problème de la modernisation des gares évoquée notamment par les cas de Saint-Lazare et Montparnasse à Paris.

Le vaste thème des rapports entre la gare et la ville, c'est-à-dire le rôle du chemin de fer dans le développement urbain, a fait l'objet de nombreux travaux, parmi lesquels on peut citer ceux sur Angers, Metz, Nancy, Bordeaux, Rennes, Marseille, Arras ou Douai, et même les nouvelles gares TGV. D’une manière plus exclusive, le chemin de fer a créé ses propres villes, les agglomérations cheminotes, telles Trappes, Morcenx, Miramas ou Audun-leRoman qui ont également été analysées au travers de leur histoire ${ }^{14}$.

Les rapports entre la mobilité et les territoires, c'est en premier lieu la réalité des trafics, de marchandises comme de voyageurs, en d'autres termes: «Le chemin de fer : pour qui, pour quoi ? "Cette question a valu un certain nombre de contributions sur les politiques commerciales des compagnies de chemins de fer. Dès son premier colloque en 1988, «Les chemins de fer,

11- RHCF 7.

12- RHCF 4.

13- RHCF 5-6.

14- [54] [89]. 
l'espace et la société en France », l'AHICF a présenté quelques exemples de travaux sur les politiques commerciales de marchandises d'anciens réseaux (Ouest, Médoc) ou de la SNCF depuis sa création, mais aussi sur quelques aspects de la politique commerciales de voyageurs (trains de nuit ou grande

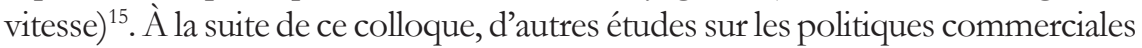
seront développées. En ce qui concerne les marchandises, on peut citer le trafic charbonnier du Nord, celui du monde agricole et en particulier des primeurs du Sud-Est ou celui des expéditions générales, ou encore le cadre dans lequel sont acheminés ces trafics, la tarification au XIX ${ }^{\mathrm{e}}$ siècle et au début du XXe siècle, ou encore le contexte réglementaire du trafic de marchandises de la SNCF depuis l'après-guerre jusqu'à la libéralisation des prix sous la pression des instances européennes ${ }^{16}$. Le rôle des embranchements particuliers a été aussi analysé, notamment dans les quartiers industriels de Lyon, ainsi que celui du bassin minier du Nord ou de la conteneurisation sur le trafic ferroviaire de fret, tout comme l'acheminement des wagons isolés ${ }^{17}$.

Au regard des trafics de voyageurs, on note des études sur le rôle du chemin de fer sur le tourisme de masse, sur les liaisons entre la France et la Grande-Bretagne, ou encore sur les trains-autos accompagnées, mais aussi sur le rôle du chemin de fer dans le développement du tourisme de masse dans la première moitié du $\mathrm{XX}^{\mathrm{e}}$ siècle, thème qui sera repris à travers la politique touristique du PLM et celle de la Compagnie du Nord, ainsi que par une étude sur la place du chemin de fer dans le tourisme en région Provence-Alpes-Côte d'Azur ${ }^{18}$.

Mais le chemin de fer a largement contribué au développement régional. Des recherches monographiques l'ont montré dans un grand nombre de régions : en Charente, dans le Trièves, dans la Vienne, en Anjou, en Vendée et dans le Lot-et-Garonne où les effets de l'implantation ferroviaire sur le développement agro-alimentaire ont été essentiels ${ }^{19}$. Il n'est pas jusqu'à l'Île-de-France où l'on se soit attaché à mettre à jour les effets économiques de l'électrification de la banlieue Saint-Lazare ou la ligne d'interconnexion des TGV.

15- RHCF HS 1.

16- RHCF HS 3 et RHCF 9.

17- [9] [81].

18- [10] [60].

19- [25] [11] [24] [65]. 
Mais si les réseaux ferrés supportent des trafics plus ou moins importants, ceux-ci s'opèrent dans un cadre économique et politique qui évolue avec le temps, ce qui a pour effet de modifier la configuration des réseaux, le plus souvent dans le sens de la contraction. Ce processus général a été analysé notamment au niveau national à travers la coordination des transports dans les années 1930, et par des études de cas régionales, notamment dans l'Ille-etVilaine, l'Indre, le Vaucluse, les Bouches-du-Rhône et le $\operatorname{Var}^{20}$. Mais, une fois ce redéploiement effectué, que faire des voies ferrées supprimées ? C'est ce que mettent au jour des études sur les voies ferrées lorraines ou la Petite Ceinture de Paris. Au stade final de l'évolution, elles peuvent être réhabilitées sous forme de chemins de fer touristiques comme le montrent notamment les exemples de la Baie de Somme ou de la Brévenne ${ }^{21}$.

\section{Pistes de recherche pour l'avenir}

Rappelons d'abord, pour éviter toute ambiguité, que l'AHICF ne fait pas elle-même de recherche mais fait faire des recherches. Le contenu de ses travaux provient des intérêts universitaires (eux-mêmes soumis aux évolutions des intérêts académiques, voire aux modes), des historiens amateurs (au meilleur sens du terme), des témoignages, des expériences des praticiens ou ex-praticiens.

Les travaux réalisés et rassemblés depuis vingt ans sous l'égide de l'AHICF ont mis en évidence la puissance et la prégnance du transport ferroviaire en France. L'histoire de l'installation d'un mode de transport, progressivement organisé en quasi-monopole économique et technique sur le territoire français, est désormais mieux connue, même si certaines zones d'ombre demeurent, notamment du côté des usages du chemin de fer mais aussi du côté de la couverture du territoire par les recherches. On observe ainsi de vastes lacunes concernant une grande partie des Alpes et des Pyrénées, le Massif central et aussi, de manière peut-être plus surprenante, le Bassin parisien hors Île-deFrance. Ces lacunes dessinent une sorte de «Désert français » et interrogent peut-être notre perception des rapports entre chemin de fer et territoire.

Quoi qu'il en soit, on a pu comprendre l'installation en France du chemin de fer comme mode de transport à part entière, au sens des habitudes.

20- [59] [95] [79] et RHCF 9.

21- Voir Etienne Auphan, "Les chemins de fer touristiques entre patrimoine et tourisme récréatif », RHCF 20-21, p. 255-288 et "L'apogée des chemins de fer secondaires en France : essai d'interprétation cartographique », RHCF 24-25, p. 24-45. 
Si l'on comprend mieux désormais comment une telle organisation s'est imposée à l'échelle nationale pour le transport ferroviaire, la question des rapports entre le chemin de fer et les autres modes de transport n'a pas encore trouvé dans les travaux de l'AHICF toutes ses réponses. Le chemin de fer a-t-il définitivement compromis l'avenir de la batellerie ? Le camionnage, l'oléoduc, le gazoduc ont-ils tué le fret ferroviaire ? Comment s'est opérée la concurrence entre le mode ferroviaire et les autres modes dans un contexte de mobilité accrue des marchandises ? Même en restant dans le mode ferroviaire, on n'a sans doute pas assez étudié les formes émergentes ou larvées qui anticipèrent les tendances à la dérèglementation. Ces formes ont existé, de manière plus ou moins cachée, semi-officielle, tolérée, marginale. Il faudrait les recenser et pousser l'investigation sur les raisons de leur émergence et de leur insuccès ou de leur échec.

Pour le transport des personnes, les questions demeurent encore plus complexes et ouvertes. La seconde moitié du XXe siècle a été marquée par une croissance très forte des mobilités. Si sur certaines liaisons particulières (lignes à grande vitesse, banlieue parisienne, peut-être aujourd'hui TER) le chemin de fer a bénéficié de cette tendance, ce sont d'autres modes de transport, l'automobile et l'avion, dont le rôle était auparavant négligeable, qui ont absorbé l'essentiel de l'accroissement des trafics. Cette évolution était-elle inéluctable du fait des technologies en présence ? A-t-elle partie liée avec la géographie particulière de la France et l'aménagement de son territoire? Ou bien résultet-elle de politiques, de choix stratégiques (en faveur du TGV par exemple) adoptés à un moment où la motorisation apparaissait comme une tendance naturelle et où le transport aérien semblait ne pouvoir être contré que sur les moyennes portées?

Le train a sans doute puissamment contribué à la fabrique du territoire français, tel qu'il s'est organisé et aménagé, en assurant une partie des navettes domicile-travail, une forte proportion des voyages d'affaires et des déplacements à grande distance pour motifs dits «personnels». Pourtant la mobilité des loisirs et du tourisme dont la croissance est depuis quelques décennies très soutenue (sinon soutenable) boude le mode ferroviaire. Comprendre les rapports entre le chemin de fer et ce type particulier de mobilité « libre » demande des investigations historiques. Le chemin de fer a-t-il été réactif par rapport aux tendances de la mobilité « choisie » ? Rappelons ici l'extraordinaire développement du tourisme de court séjour, bien avant les 35 heures (en fait depuis le début des années 1980). Ces courts séjours relèvent principalement d'un tourisme urbain, ce qui donnait toutes ses chances au mode ferroviaire. Pourquoi ce 
vaste créneau n'a-t-il pas été investi par le chemin de fer comme il aurait pu l'être dans un pays comme la France? Qu'est-il advenu des initiatives prises ici ou là (trains-auto, trains de pèlerinage, chemins de fer touristiques) ? Disons seulement un mot des trains-autos. L'AHICF n'a pas ignoré la question mais sous forme monographique ${ }^{22}$. Resterait à comprendre quel est le sens du mouvement. S'agit-il d'une intermodalité tendancielle, ou d'une forme moribonde de transport ? Difficile de le savoir. Alors que l'on annonce régulièrement la fin des TAC, Narbonne reçoit encore aujourd'hui bon mal an deux TAC par jour de semaine et quatre par jour de week-end. Y a-t-il eu vraie concurrence avec les autres modes ? Le train a certes fait beaucoup dans le passé, à la fois en faveur d'un tourisme élitiste et pour le transport de masse vers des stations de vacances. Mais la généralisation des loisirs et du tourisme à la carte relevaitelle encore du mode ferroviaire ou lui échappait-elle par nature?

Les colloques qu'organise l'AHICF, les recueils d'information qu'elle commande, les travaux universitaires qu'elle soutient ne sauraient évidemment apporter des réponses définitives à des questions aussi générales. Les vingt années de l'Association ont permis de constater que les recherches portent toujours sur telle technique particulière, tel territoire, tel segment de population. Elles s'appuient sur telle base de données ou telle source d'archives. Les sujets peuvent paraître un peu minces, les résultats incertains. Mais la connaissance progresse à petits pas. L'essentiel est d'aller dans la bonne direction.

22- Raoul Balso, «Évolution du service des trains auto accompagnées sur la SNCF (1957-1987) », RHCF 9, p. 113-129 et [60]. 
